03;04

\title{
Исследование влияния параметров импульсного разряда на порождаемые в сверхзвуковом пограничном слое локализованные возмущения
}

\author{
(C) А.А. Яцких ${ }^{1,2}$, Ю.Г. Ермолаев ${ }^{1}$, А.Д. Косинов ${ }^{1,2}$, Н.В. Семенов ${ }^{1}$ \\ ${ }^{1}$ Институт теоретической и прикладной механики им. С.А. Христиановича СО РАН, Новосибирск, Россия \\ ${ }^{2}$ Новосибирский государственный университет, Новосибирск, Россия \\ E-mail: yatskikh@itam.nsc.ru
}

Поступило в Редакцию 3 декабря 2018 г.

В окончательной редакции 3 декабря 2018 г.

Принято к публикации 10 декабря 2018г.

\begin{abstract}
Представлены результаты экспериментальных исследований влияния длительности и силы тока импульсного тлеющего разряда на генерацию локализованных возмущений в сверхзвуковом пограничном слое плоской пластины при числах Маха набегающего потока $\mathrm{M}=3$ и 4. Измерения пульсаций потока производились термоанемометром. Обнаружено, что увеличение длительности импульсного воздействия приводит к увеличению амплитуды и временны́х масштабов генерируемых в пограничном слое возмущений. Увеличение силы тока импульсного разряда приводит к увеличению амплитуды порождаемых пульсаций. Оценены скорости распространения в пограничном слое локализованных возмущений при различных числах Маха набегающего потока.
\end{abstract}

DOI: 10.21883/PJTF.2019.05.47402.17618

В настоящее время методы активного управления различными аэродинамическими течениями с помощью плазменных актуаторов интенсивно развиваются [1-7]. Основными примуществами воздействия на течения с помощью газоразрядных систем являются простота конструкции и отсутствие движущихся частей. Помимо прикладных исследований плазменные актуаторы используются в качестве генераторов искусственных контролируемых возмущений при изучении ламинарнотурбулентного перехода высокоскоростных пограничных слоев. Синхронизируя по времени измерения с моментом возбуждения искусственных пульсаций, можно выделить их из фоновых естественных возмущений пограничного слоя, что позволяет получать детальные данные об эволюции возмущений. Так, с помощью генерации разрядом периодических контролируемых пульсаций экстремально подтверждена линейная теория гидродинамической неустойчивости для сверхзвукового пограничного слоя пластины [8] и скользящего крыла [9].

Для изучения поздних стадий ламинарно-турбулентного перехода перспективным является исследование эволюции локализованных во времени и пространстве возмущений [10]. Для случаев высокоскоростных пограничных слоев локализованные возмущения могут быть возбуждены с помощью импульсного разряда $[11,12]$. В ИТПМ СО РАН подход контролируемых локализованных возмущений используется и активно развивается. В работах [13-15] локализованные возмущения генерируются в пограничном слое с помощью импульсного разряда, зажигающегося между двумя электродами, выведенными на поверхность экспериментальной модели. Исследования [14] показали, что энергия и длительность импульсного разряда влияют на пространственные масштабы и амплитуду генерируемых в пограничном слое возмущений. Для развития методики возбуждения контролируемых локализованных возмущений необходимы систематизированные данные по влиянию на амплитуду и простраственные масштабы генерируемых возмущений таких параметров, как сила тока импульсного разряда и его длительность.

В работе [16] проведены экспериментальные исследования влияния длительности импульсного разряда на генерируемые в пограничном слое локализованные возмущения при числе Маха $\mathrm{M}=2$. Настоящая работа посвящена исследованию влияния длительности и силы тока импульсного тлеющего разряда (ток разряда менее $10 \mathrm{~mA}$ ) на амплитуду и временны́е масштабы локализованных возмущений, генерируемых им в пограничном слое плоской пластины при числах Маха $\mathrm{M=3}$ и 4. В работе анализируются как новые экспериментальные данные, так и полученные ранее в [16].

Эксперименты выполнялись в сверхзвуковой малотурбулентной аэродинамической трубе (АДТ) Т-325 ИТПМ СО РАН. Эксперименты при $\mathrm{M}=3$ были проведены при значении единичного числа Рейнольдса $\mathrm{Re}_{1}=8.8 \cdot 10^{6} \mathrm{~m}^{-1}$, а при $\mathrm{M}=4$ - при значении $\mathrm{Re}_{1}=9.7 \cdot 10^{6} \mathrm{~m}^{-1}$. Во всех экспериментах температура торможения составляла $T_{0}=292-296 \mathrm{~K}$.

В качестве экспериментальной модели использовалась плоская пластина с острой передней кромкой, установленная под нулевым углом атаки. Описание модели приведено в [16]. Модель оборудована источником контролируемых возмущений: двумя медными электродами, расположенными в центральной области пластины. 


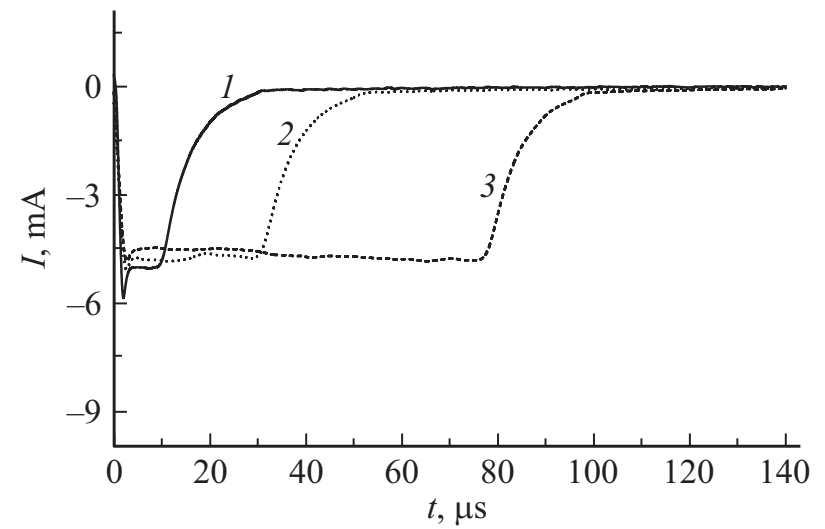

Рис. 1. Временнб́е зависимости силы тока импульсного разряда при длительности управляющего сигнала $\tau \approx 5(1)$, 25 (2) и $75 \mu \mathrm{s}(3)$.

Электроды отделены друг от друга и от модели керамическим изолятором. Изолятор и электроды выведены на поверхность модели и отшлифованы заподлицо. Центры электродов находятся на линии, параллельной направлению набегающего потока. Диаметры электродов $0.9 \mathrm{~mm}$, минимальное расстояние между ними $\sim 0.9 \mathrm{~mm}$. Центр первого электрода располагался на расстоянии $x \approx 27 \mathrm{~mm}$ от передней кромки. Продольная координата $x$ отсчитывалась от передней кромки модели.

Для подробных исследований влияния параметров импульсного разряда на возбуждаемые локализованные возмушения сверхзвукового пограничного слоя использовалась схема зажигания импульсного тлеющего разряда, позволяющая контролировать длительность импульсного разряда, его силу тока и частоту зажигания. Работа схемы основана на модуляции постоянного высокого напряжения с помощью быстродействующего высоковольтного ключа. Схема зажигания позволяет генерировать импульсы высокого напряжения амплитудой до $3 \mathrm{kV}$ и длительностью от $5 \mu \mathrm{s}$. Управление высоковольтным ключом было синхронизировано с измерительным комплексом АДТ-325.

На рис. 1 показаны осциллограммы силы тока при различных значениях длительности управляющего сигнала: 5, 25 и $75 \mu \mathrm{s}$. Представлены данные, полученные при зажигании разряда на поверхности модели пластины при числе Маха набегающего потока $\mathrm{M}=4$. В нулевой момент времени подается сигнал на открытие высоковольтного ключа. В течение приблизительно $4 \mu \mathrm{s}$ наблюдается зарядка емкости разрядника. Пробой межэлектродного промежутка сопровождается скачком тока. После зажигания наблюдается установившееся горение разряда, при котором ток и напряжение слабо изменяются. Через определенное время $(5,25$ или $75 \mu \mathrm{s})$ на ключ подается сигнал на закрытие, и разряд гасится, на что указывает изменение силы тока до нуля.

Для измерения возмущений использовался термоанемометр постоянного сопротивления. Датчик тер- моанемометра изготавливался из вольфрамовой нити диаметром $10 \mu \mathrm{m}$. Величина перегрева нити датчика устанавливалась равной 0.8, а измеренные возмущения на 95\% состояли из пульсаций массового расхода [17]. С помощью 12-разрядного аналого-цифрового преобразователя (АЦП) записывался полный сигнал термоанемометра. Частота дискретизации АЦП составляла $1.25 \mathrm{MHz}$. Записанный сигнал разделялся на пульсационную $e^{\prime}$ и постоянную $E$ составляющие. Постоянная составляющая сигнала $E$ высчитывалась по части осциллограммы, в которой не было возмущений от разряда. Нормированные на локальное среднее течение пульсации массового расхода $m^{\prime}$ вычислялись как отношение пульсационного сигнала к постоянной составляющей с учетом коэффициента чувствительности $S_{\rho U}=0.25 \pm 0.02[17]$ :

$$
m^{\prime}(t)=(\rho U)^{\prime} / \overline{\rho U} \approx e^{\prime}(t) /\left(S_{\rho U} E\right),
$$

где $\rho, U-$ плотность и продольная скорость потока.

Для выделения искусственных возмущений из фоновых естественных флуктуаций пограничного слоя измерения проводились синхронно по времени с зажиганием разряда. В каждой точке записывались 320 реализаций сигнала, а обработка данных состояла из синхронного осреднения 320 осциллограмм (аналогично измерениям в [14]).

Измерения проводились в центральной области возмущений $(z=0 \mathrm{~mm})$, возбуждаемых разрядом. Датчик термоанемометра устанавливался в области пограничного слоя, в которой среднее течение оставалось постоянным: $\rho U \approx 0.7 \rho U_{\infty}$, что соответствует области с максимальным уровнем естественных пульсаций.

На рис. 2, $a$ и $b$ представлены результаты, полученные при значениях числа Маха набегающего потока $\mathrm{M}=3$ и 4 соответственно. Показаны осциллограммы локализованных возмущений, генерируемых импульсным разрядом различной длительности. Измерения проведены в пограничном слое при значениях продольной координаты $x=60$ и $100 \mathrm{~mm}$.

При увеличении длительности импульсного разряда растут временны́е масштабы и амплитуда генерируемых возмущений. При этом нарастание передних фронтов волновых пакетов от разряда с различной длительностью происходит по одному закону. По представленным данным проведены оценки скорости распространения волновых пакетов по времени регистрации максимального отклонения от среднего течения. Получено, что скорость распространения локализованных возмущений составляет приблизительно $65 \%$ от скорости набегающего потока в случае $\mathrm{M}=3$, а для $\mathrm{M}=4-78 \%$. В исследованиях [16] было получено, что при $\mathrm{M}=2$ локализованные возмущения распространяются со скоростью приблизительно 60\% от скорости набегающего потока. Полученные значения скоростей распространения локализованных возмущений близки к оценке по соотношению $(\mathrm{M}-1) / \mathrm{M}$, что соответствует локальным скоростям на уровне критического слоя. 

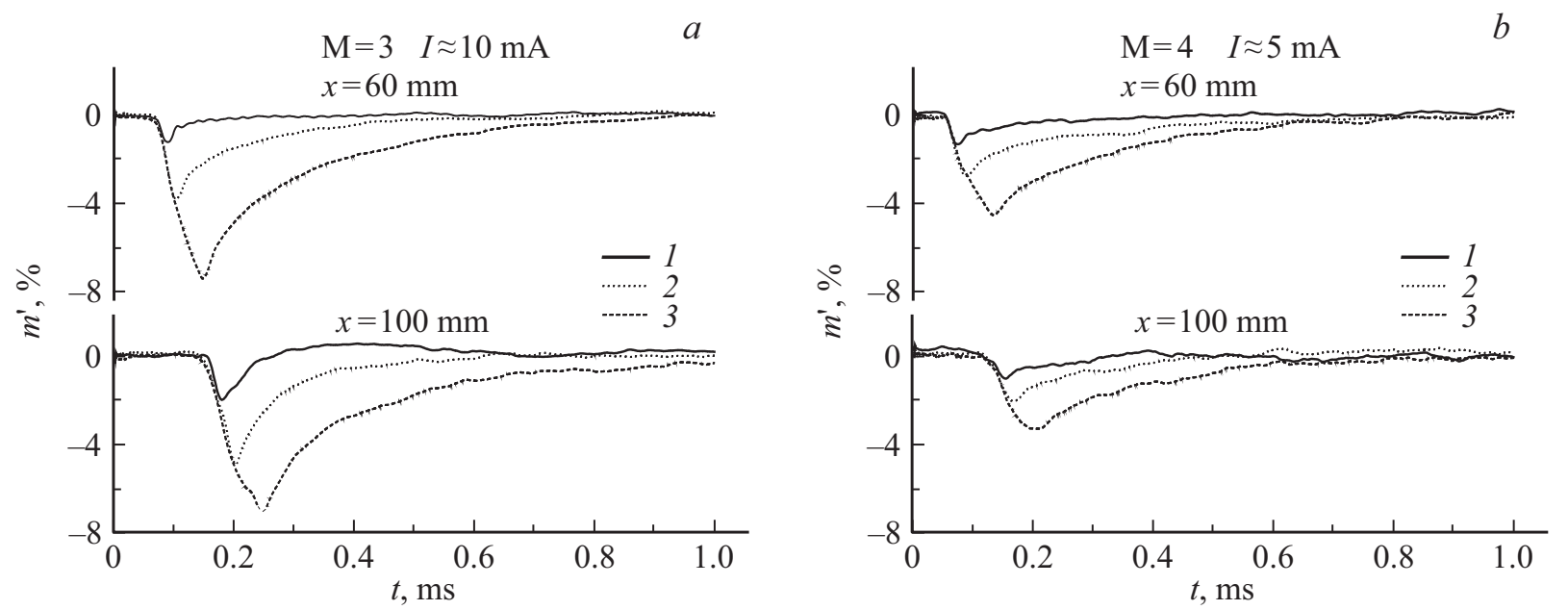

Рис. 2. Локализованные возмущения от импульсного разряда различной длительности при числе Мaха $\mathrm{M}=3(a)$ и $4(b) . \tau \approx 5$ (1), 25 (2) и $75 \mu \mathrm{s}(3)$.

$a$

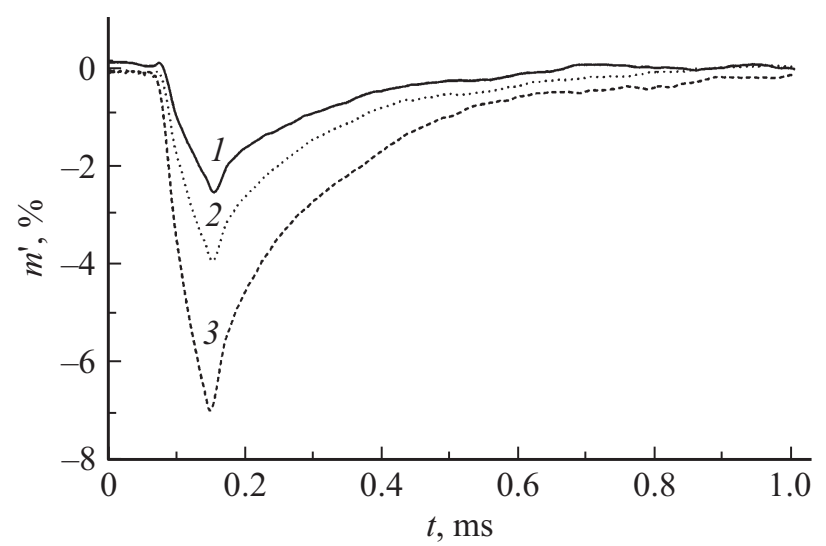

$b$

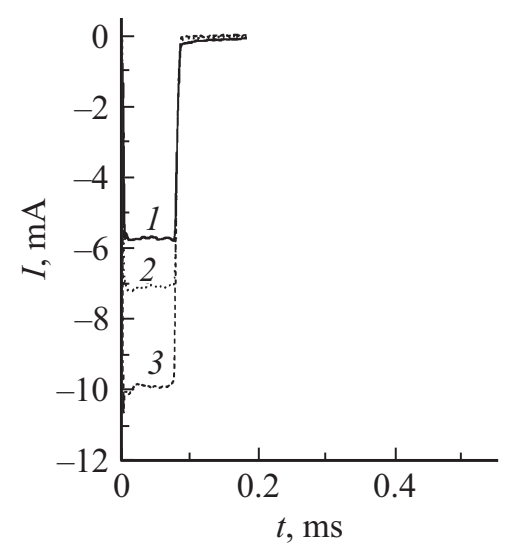

Рис. 3. Локализованные возмущения от импульсного разряда при различной силе тока. $\mathrm{M=}$.

На рис. 3 представлены результаты исследований влияния силы тока импульсного разряда на генерируемые им локализованные возмущения. На рис. 3, $a$ показаны осциллограммы локализованных возмущений, генерируемых импульсным разрядом с различной силой тока. На рис. $3, b$ представлены соответствующие зависимости силы тока разряда. Измерения проводились при числе Маха набегающего потока $\mathrm{M}=3$ в пограничном слое на расстоянии $60 \mathrm{~mm}$ от передней кромки. Длительность горения импульсного разряда оставалась постоянной и была приблизительно равна $75 \mu \mathrm{s}$.

Длительность нарастающей части локализованного возмушения близка ко времени горения разряда. При увеличении силы тока, протекающего через разряд, амплитуда возмущений увеличивается. При этом интенсивность нарастания также зависит от силы тока разряда. Затухающая часть регистрируемых возмущений значительно более длительная. Экспериментальные исследования, проведенные при числе Маха набегающего потока $\mathrm{M}=4$, показывают аналогичные результаты.
На основе результатов экспериментальных исследований можно сделать следующие выводы.

1. При числах Маха набегающего потока $\mathrm{M}=2,3$ и 4 в пограничном слое плоской пластины локализованные возмущения распространяются вниз по потоку со скоростью, близкой к локальным скоростям в критическом слое.

2. Увеличение длительности воздействия на сверхзвуковой пограничный слой приводит к увеличению временны́х масштабов и амплитуды возбуждаемых локализованных возмущений.

3. При увеличении силы тока импульсного разряда амплитуда и интенсивность нарастания фронтов генерируемых локализованных возмушений сверхзвукового пограничного слоя возрастают.

Работа выполнена в рамках базового государственного задания (номер госрегистрации AAAA-A17-117030610125-7). 


\section{Список литературы}

[1] Moreau E. // J. Phys. D: Appl. Phys. 2007. V. 40. N 3. P. $605-$ 636. DOI: $10.1088 / 0022-3727 / 40 / 3 / \mathrm{S} 01$

[2] Post M.L., Corke T.C. // AIAA J. 2004. V. 42. N 11. P. $2177-$ 2184. DOI: $10.2514 / 1.2929$

[3] Алешин Б.С., Курячий А.П., Ребров И.Е., Хомич В.Ю., Чернышев С.Л., Ямщиков В.А. // Письма в ЖТФ. 2017. T. 43. B. 1. C. 45-52.

[4] Лапушкина Т.А., Ерофеев А.В. // Письма в ЖТФ. 2017. T. 43. B. 5. C. 17-23.

[5] Поливанов П.А., Сидоренко А.А. // Письма в ЖТФ. 2018. T. 44. B. 18. C. $60-68$.

[6] Знаменская И.А., Сысоев Н.Н., Цзинь Ц. // Письма в ЖТФ. 2013. Т. 39. В. 9. С. 28-33.

[7] Moralev I., Sherbakova V., Selivonin I., Bityurin V.,Ustinov M. // Int. J. Heat Mass Transfer. 2018. V. 116. P. 13261340.DOI: 10.1016/j.ijheatmasstransfer.2017.09.121

[8] Kosinov A.D., Maslov A.A., Shevelkov S.G. // J. Fluid Mech. 1990. V. 219. P. 621-633. DOI: 10.1017/S0022112090003111

[9] Kosinov A.D., Kolosov G.L., Semionov N.V., Yermolaev Y.G. // Phys. Fluids. 2016. V. 28. P. 064101.DOI: 10.1063/1.4952999

[10] Катасонов М.М., Козлов В.В., Никитин Н.В., Сбоев Д.С. Возникновение и развитие локализованных возмущений в круглой трубе и пограничном слое. Новосибирск: НГУ, 2014. $222 \mathrm{c}$.

[11] Casper K.M., Beresh S.J., Schneider S.P. // J. Fluid Mech. 2014. V. 756. P. 1058-1091. DOI: $10.1017 / \mathrm{jfm} .2014 .475$

[12] Casper K.M. Fluid-structure interactions on a slender cone under quiet flow conditions at Mach $6 / /$ 58th AIAA/ASCE/AHS/ ASC Structures, Structural Dynamics, and Materials Conf. American Institute of Aeronautics and Astronautics, 2017. AIAA paper N 2017-0402. DOI: 10.2514/ 6.2017-0402

[13] Яцких А.А., Ермолаев Ю.Г., Косинов А.Д., Семенов Н.В. // Теплофизика и аэромеханика. 2015. Т. 22. В. 1. С. 17-28.

[14] Yatskikh A.A., Yermolaev Y.G., Kosinov A.D., Semionov N.V. // J. Visualization. 2017. V. 20. N 3. P. 549-557. DOI: $10.1007 / \mathrm{s} 12650-016-0414-2$

[15] Yatskikh A., Yermolaev Y., Kosinov A., Semionov N., Semenov A. // Proc. Inst. Mech. Eng. G: J. Aerospace Eng. First Publ. July 12, 2018. DOI: $10.1177 / 0954410018787120$

[16] Яцких А.А., Семенов А.Н., Ермолаев Ю.Г., Косинов А.Д., Семенов Н.В. // Сиб. физ. журн. 2017. Т. 12. В. 3. С. 41-48.

[17] Kosinov A.D., Semionov N.V., Yermolaev Yu.G. Disturbances in test section of T-325 supersonic wind tunnel ITAM SB RAS. Preprint N 6-99. Novosibirsk: ITAM SB RAS, 1999. $24 \mathrm{p}$. 